\title{
A digital twin framework for improving energy efficiency and occupant comfort in public and commercial buildings
}

Anders Clausen ${ }^{1 *}$, Krzysztof Arendt $^{1}$, Aslak Johansen $^{2}$, Fisayo Caleb Sangogboye ${ }^{1}$, Mikkel Baun Kjærgaard², Christian T. Veje ${ }^{1}$ and Bo Nørregaard Jørgensen ${ }^{1}$

From 1st Energy Informatics.Academy Conference Asia

Beijing, China . 29-30 May 2021

\footnotetext{
* Correspondence: ancla@mmmi. sdu.dk

${ }^{1}$ Center for Energy Informatics, Maersk Mc-Kinney Moller Institute, University of Southern Denmark, Campusvej 55, 5230 Odense M, Denmark

Full list of author information is available at the end of the article
}

\begin{abstract}
Model Predictive Control (MPC) can be used in the context of building automation to improve energy efficiency and occupant comfort.

Ideally, the MPC algorithm should consider current- and planned usage of the controlled environment along with initial state and weather forecast to plan for optimal comfort and energy efficiency.

This implies the need for an MPC application which 1) considers multiple objectives, 2) can draw on multiple data sources, and 3) provides an approach to effectively integrate against heterogeneous building automation systems to make the approach reusable across different installations.

To this end, this paper presents a design and implementation of a framework for digital twins for buildings in which the controlled environments are represented as digital entities. In this framework, digital twins constitute parametrized models which are integrated into a generic control algorithm that uses data on weather forecasts, current- and planned occupancy as well as the current state of the controlled environment to perform MPC. This data is accessed through a generic data layer to enable uniform data access. This enables the framework to switch seamlessly between simulation and real-life applications and reduces the barrier towards reusing the application in a different control environment. We demonstrate an application of the digital twin framework on a case study at the University of Southern Denmark where a digital twin has been used to control heating and ventilation.

From the case study, we observe that we can switch from rule-based control to model predictive control with no immediate adverse effects towards comfort or energy consumption. We also identify the potential for an increase in energy efficiency, as well as introduce the possibility of planning energy consumption against local electricity production or market conditions, while maintaining occupant comfort.
\end{abstract}

Keywords: Model predictive control, Genetic algorithm, Software framework 


\section{Introduction}

Buildings are responsible for approximately $40 \%$ of the total energy consumption in industrialized countries (Cao et al. 2016a) and they, therefore, present large opportunities but also barriers (Ma et al. 2016) for improving energy efficiency by advancing building intelligence (Jørgensen et al. 2015) Buildings are part of complex ecosystems where the primary uses of the buildings are more important than their energy use. This is for instance the case for commercial buildings like retail stores (Ma et al. 2017a) and hospitals (Billanes et al. 2018).

The advancement of building intelligence is concerned with plugged-in devices as well as various building instrumentation devices - including sensors, actuators, and controllers - that enable energy-efficient building automation while ensuring the comfort of building occupants (Billanes et al. 2017; Lazarova-Molnar et al. 2015).

The indoor building environment might react slowly to changes in control, potentially leading to temporal discomfort periods. Traditionally, a solution was to maintain relatively stable indoor conditions through constant setpoints. This approach is, however, energy inefficient. Furthermore, the surge of renewable energy sources puts pressure on buildings to allow more energy flexibility, e.g. from Demand Response programs (Ma et al. 2017b; Kjærgaard et al. 2016a). Model Predictive Control (MPC) is well adapted to these new requirements (Bianchini et al. 2016).

Therefore, there are ongoing efforts, both in the industry and research, to develop methods for improved management of energy in buildings (Al-Ghaili et al. 2021). One of the approaches recently gaining more momentum both in research and industry is MPC (Drgoňa et al. 2020). MPC represents a family of control strategies directly dependent on physical models (Garcia et al. 1989a). The use of models, together with forecasted model inputs, enables us to consider the system dynamics in control planning. This feature is especially attractive for high inertia systems, such as buildings with high thermal mass (Li and Malkawi 2016a).

The practical application of MPC and a commercial adaptation of such an approach requires several challenges to be addressed.

For one, MPC in building automation requires an interface towards the physical installations in the targeted building(s). These installations constitute sensors and actuators which are configured, installed, and deployed depending on the physical properties of the building and the building automation degree used on the building. This means that installations vary significantly between buildings.

Further, the successful application of MPC requires a model able to predict building response to actuation. This model must take into account the physical properties of the building as well as weather predictions and occupancy predictions. These properties are again highly heterogeneous between buildings.

Finally, a vast majority of MPC frameworks reported in literature define the associated optimization problem in terms of constrained minimization, using a single cost function potentially containing multiple normalized objectives and a set of constraints, e.g. temperature limits, system capacity limits, etc. (Bianchini et al. 2016; De Coninck and Helsen 2016a). Although this approach proved to be successful, with saving from $10 \%$ to $40 \%$ reported in several case studies (De Coninck and Helsen 2016a; Dong and Lam 2014), it suffers from an inflexible definition of the cost function that does not map well to the problem domain. In these approaches, weights are used to balance 
contributions from each objective in the cost function. The value of these weights is found through tuning where values are essentially adjusted until the output of the cost function makes sense. Changing the priorities of, adding, or removing objectives might require re-tuning of the weights in the cost function, and output is never guaranteed to be stable outside of the test set. This problem is amplified when considering the heterogeneous nature of buildings: A cost function tuned to a particular building cannot be directly transferred to another building with another underlying model.

To overcome these challenges, this paper presents a digital twin framework for MPCbased building automation. The digital twin framework enables the creation of a virtual replica of a building's physical environment and helps facility managers to gain new operation insights, optimize energy use, and monitor building performance.

The main parts of the framework are a) a generic Zone Control Application based on Controleum (Sørensen and Jørgensen 2017), an object-oriented optimization framework based on a Genetic Algorithm (GA), which in this paper has been configured towards building automation b) a parametrized grey-box zone model used by the GA to calculate consequences of specific actuation in a given environmental context and c) a data layer which uses sMAP (Dawson-Haggerty et al. 2010) to provide uniform and parametrized access to data sources including building sensors and actuators as well as weather forecasts and other sources of predictive information. In the case of this paper, this predictive information includes an occupancy prediction algorithm, OccuRE (Kjærgaard et al. 2016b; Sangogboye et al. 2017), which predicts occupant behavior for our case building.

The use of Controleum as a foundation for the Zone Control Application means that cost functions are defined on a per-objective basis. During optimization, a Pareto set of solutions are generated, and after termination, a ranked order selection mechanism can select the best solution considering the individual importance of the defined objectives (Clausen et al. 2020a). This means that no global cost function or weights are needed.

The properties combined imply that the framework is generic and reusable across different controlled environments.

To illustrate the viability of our digital twin framework we applied it to a lecture room (U182) in one of the two Smart Building Living Labs at the University of Southern Denmark. A detailed presentation of the Smart Building Living Labs is available in (Jradi et al. 2017a; Jradi et al. 2017b). We constructed a digital twin of the lecture room and used it to control heating and ventilation in the room during a normal day of operation.

The results of the experiment suggest that our digital twin approach is comparable in performance to regular operation (where rule-based control is used) concerning comfort. We also see a change in the behavior of the building automation actuators which shows a potential for an increase in energy efficiency. Switching to an MPC-based approach also introduces the possibility of planning energy consumption against local electricity production or market conditions, while maintaining occupant comfort.

The rest of the paper is structured as follows. The section "A DIGITAL TWIN FRAMEWORK FOR BUILDINGS” presents our Digital Twin Framework and discusses its constituent parts. The section "EXPERIMENTAL CASE" explains how our digital twin concept has been applied to our Smart Building Living Lab at the University of Southern Denmark campus in Odense. The section "EXPERIMENTS "presents the 
results and the section "DISCUSSION" discusses the observations that we made from the data gathered during our experiments. Finally, the section ".

CONCLUSION" concludes on the findings of the paper.

\section{A digital twin framework for buildings}

This section presents the Digital Twin Framework design and its constituent components including, OccuRE (Kjærgaard et al. 2016b; Sangogboye et al. 2017), an occupancy prediction algorithm, and a Zone Control Application based on Controleum, which uses a generic zone model, developed as part of this paper.

\section{Digital twin framework design}

Figure 1 shows an overview of the components in the digital twin. The architecture follows the BOSS principles of reusing general-purpose operating system concepts (Dawson-Haggerty et al. 2013).

At the top of the setup in Fig. 1 OccuRE (Kjærgaard et al. 2016b; Sangogboye et al. 2017) and the Zone Control Application reside. Both of these components are described in the following sections.

At the center of the setup is an sMAP bus (Dawson-Haggerty et al. 2010) as shown in Fig. 1. sMAP is a publish-subscribe substrate. By acting as a narrow waist - where it is used as a common transport layer between all components - sMAP becomes the common protocol. This provides a uniform interface for drivers and applications to communicate through. An archiver is used to store historical records of all data that passes through sMAP and exposes an interface for look-up against these data, thus providing applications with the opportunity to get information on the current state of a controlled environment (including current values for sensors and actuators) as well as predictions associated with the controlled environment i.e. predictions for local weather conditions and occupancy predictions.

The logic that interfaces the building instrumentation devices is referred to as drivers. The instrumentation devices shown in Fig. 1 can be either building instrumentation

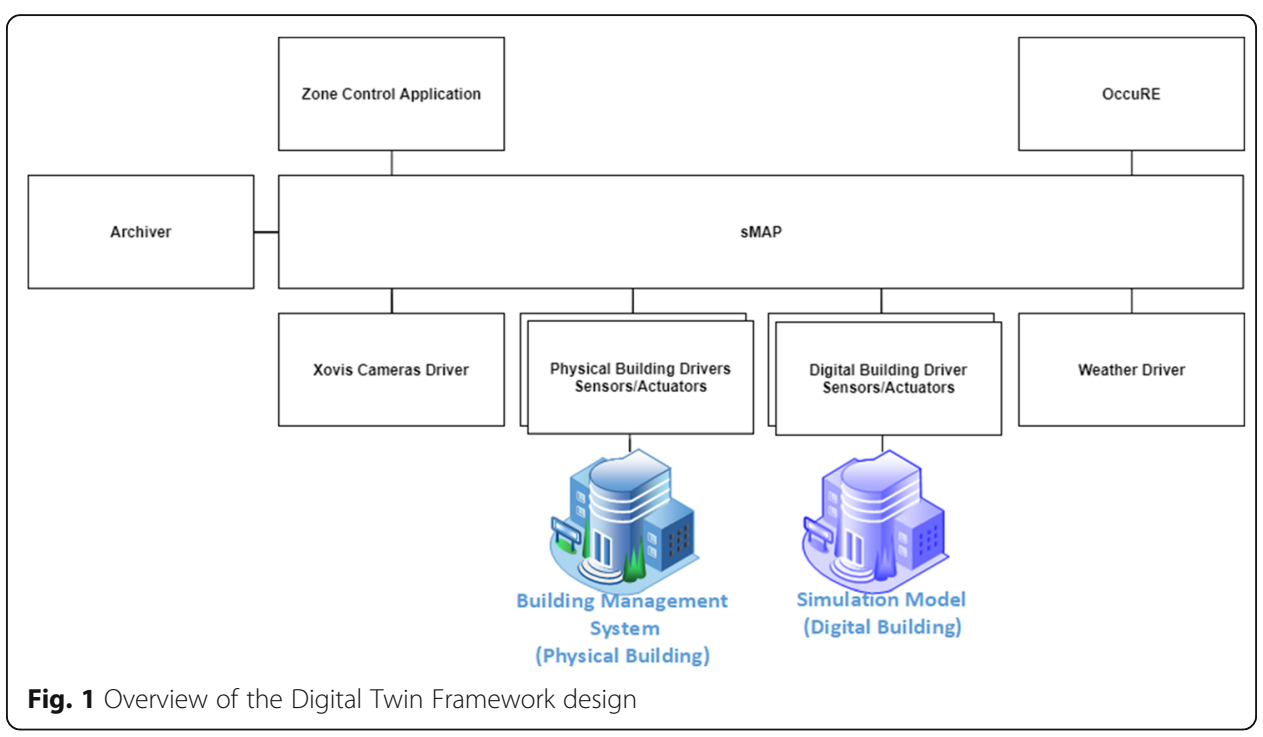


devices (in the physical building) or simulated building instrumentation devices (in the digital building). The hardware entities publish time-series data to the sMAP bus when data has been read from sensors or external services and subscribe to time series data from sMAP to provide new values to actuators in the controlled environment. In the setup employed a total of 6 drivers were used. One driver was responsible for interfacing with cameras used to register occupants, one was responsible for interfacing with an external weather forecast provider and the remaining drivers were responsible for reading sensory values- and writing actuation to building instrumentation.

\section{OccuRE - occupancy prediction}

The Zone Control Application in the proposed digital twin framework makes use of occupancy count and prediction in its optimization.

To enable estimation of occupancy behavior in the controlled environment that we consider in this paper, dedicated and pervasive sensors for extracting the non-adaptive occupant behaviors, which include the occupancy presence, counts, and locations, were used. In our case environment, the presence and counts of occupants are extracted using dedicated PIR sensors and counting camera sensors respectively.

The information from these sensors is fed into OccuRE - a stream-based occupancy platform for provisioning occupancy information in multiple spatial and temporal resolutions (Kjærgaard et al. 2016b). OccuRE includes a technology-agnostic API, the OccuRE reasoning engine, and the OccuRE building instrumentation device drivers. Figure 2 shows the components and how they are organized towards each other. The API facilitates a two-way communication procedure for requesting and provisioning occupancy information and it enables high portability of occupancy applications. The reasoning engine includes a repository of deployed occupancy estimation strategies that are selected dynamically at runtime for computing occupancy information. Lastly, the building instrumentation drivers facilitate the extraction of occupancy data from

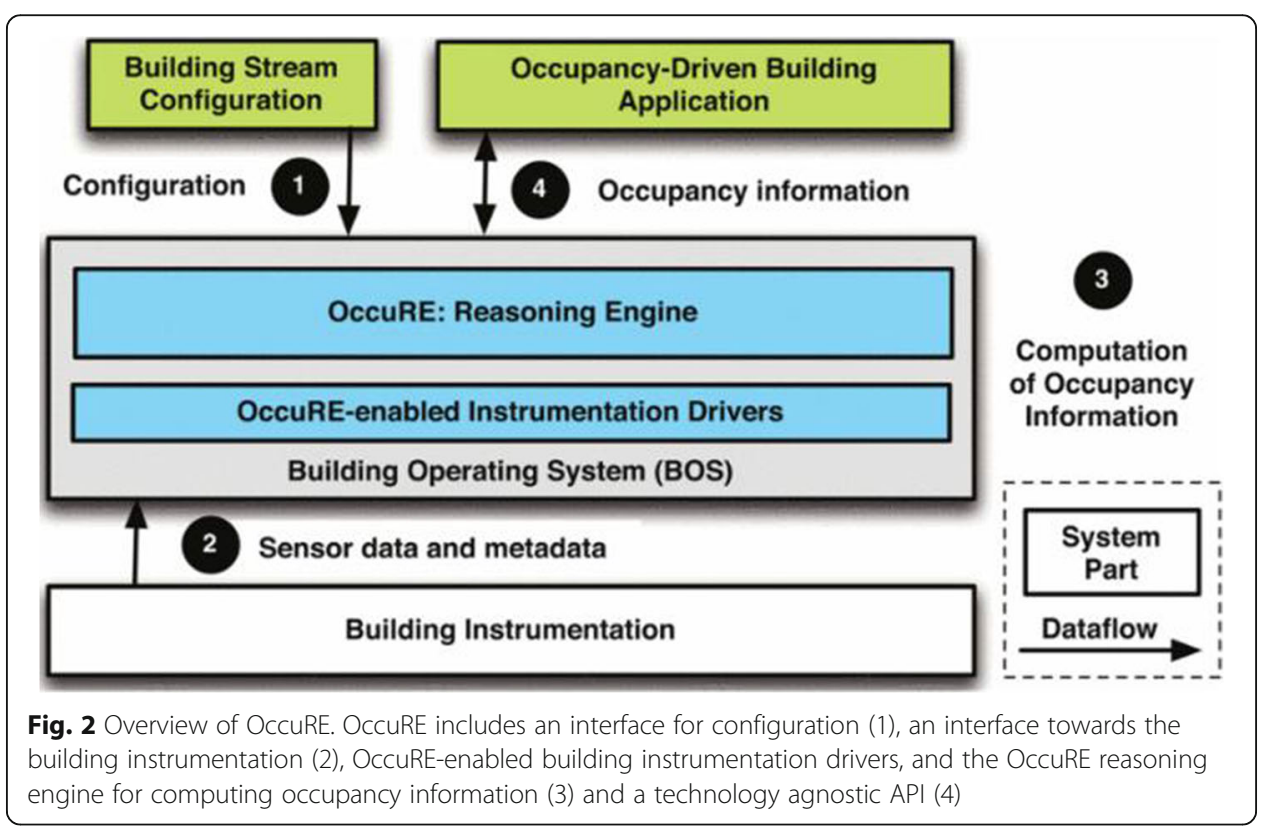


deployed sensors as well as metadata associated with the sensors. This metadata constitutes spatial and temporal data as well as information on the sensor type, the data type, and format of the sensor, and the quality of the data.

\section{Zone control application}

The purpose of the Zone Control Application is to find sets of setpoints for all controlled actuators which - when applied - satisfies comfort and energy efficiency targets in the controlled environment. We use a multi-objective optimization approach where setpoints for the controlled actuators are defined as optimization variables and targets for user comfort and energy efficiency becomes objectives.

The Zone Control Application uses a generic GA framework named Controleum (Sørensen and Jørgensen 2017) to solve the multi-objective optimization problem. The GA framework is used to generate a Pareto set of candidate solutions, where each solution contains one set of actuation points for each of the controlled actuators. Post-optimization the framework applies social welfare criteria to select a final solution from the Pareto set (Clausen et al. 2020a). Here, the use of a GA enables a global optimization over the entire Pareto set independent from the structure of objective functions (Marler and Arora 2004). The downside to this approach is that it is computationally expensive. However, the dynamic properties of the system we consider in this case study - CO2- and temperature levels in controlled zones - are comparably slow, allowing us to use several minutes to optimize in between actuations.

The Zone Control Application fetches information on the current zone state, weather forecast, and occupancy prediction from sMAP before initializing a run of the optimization. This data is used during optimization along with the actuation values generated by the GA to calculate context-specific consequences of those actuation values. The calculation of consequences is done by the generic Zone Model for each set of setpoints generated by the GA.

The model in the Zone Model component is based on the gray-box modeling paradigm, in which most only the most fundamental physical relationships between inputs, states, and outputs are included. Since the model is physically incomplete, it still relies on calibration based on measured data, although this reliance is weaker than in the case of machine learning models (Arendt et al. 2018a). Typically, measurements from only a few recent days are required to calibrate the model. The same model can be calibrated to represent different zones in a building. The calibration is performed with the ModestPy package (Arendt et al. 2018b), using multi-step optimization. Firstly, the approximate parameters are found using a GA; secondly, a gradient-based method is employed to fine-tune the parameters.

The model takes into account solar heat gains, conductive and convective heat loss through the thermal envelope, infiltration, occupancy heat and $\mathrm{CO} 2$ gains, radiator heat gains, and ventilation airflow and its influence on indoor energy and $\mathrm{CO} 2$ balance. The model is piecewise linear; the nonlinearities are only due: (1) to PI controller limits (output signals can vary from 0 to 1 ), (2) occupancy heat gains which are piecewise linearly dependent on indoor temperature, and (3) due to the ventilation supply air temperature which is defined as the maximum from the outdoor temperature and 
ventilation air temperature setpoint (because there is no mechanical cooling in the HVAC system).

The model is supplied with several inputs, as seen from Fig. 3. The Zone Control Application supplied values for "CO2stp" and "Tstp" which were generated by the GA, "Tvestp" was fixed configured with a fixed value since this reflects real life cases as the temperature of the air in the ventilation system is not changed once the system is in operation, "occ" was supplied from OccuRE and "solrad" and "Tout" is supplied from the Weather Driver depicted in Fig. 1.

The output of the Zone Model is used by objectives in the Zone Control Application to evaluate suggestions for actuation values. The Zone Control Application implements a total of 5 objectives that map to specific domain goals and which can make a context-aware evaluation of solutions with actuation suggestions by considering the output generated by the generic zone model from the corresponding solutions.

As the Controleum framework is an object-oriented software framework, each objective is modeled as a first-class abstraction. This means that objectives directly map to real-life goals for the environment, and thus can be identified based on the problem

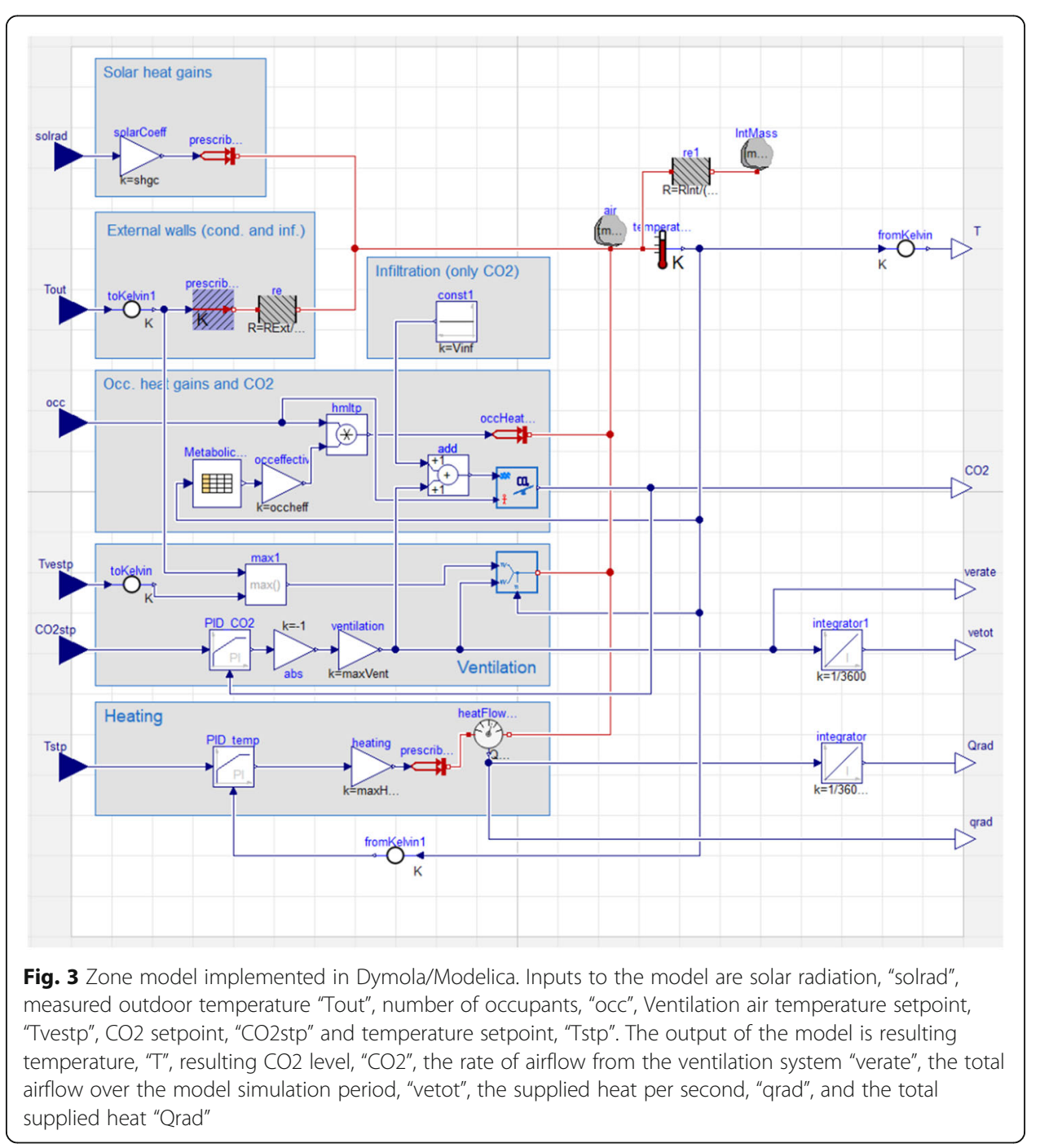


domain. To summarize, the goal of the Zone Control Application is to control actuators in the environment to create a response which 1) ensures a sound indoor climate concerning $\mathrm{CO} 2$ and temperature and 2) does so in an energy-efficient way.

From 1) we have identified the need for three objectives. An objective, the CO2 Constraint Objective, should ensure that the allowable threshold for $\mathrm{CO} 2$ inside the controlled zone does not exceed some predefined limit. Further, the temperature should be kept in an allowable temperature range which in the case of the Zone Control Application is expressed through an Upper Comfort Temperature Objective and a Lower Comfort Temperature Objective. The exact values for the $\mathrm{CO} 2$ threshold as well as the Upper- and Lower Comfort Temperature values are instance specific to accommodate the fact such values depend on the use of the controlled environment and local work environment regulations.

From 2) we identified the need for another two objectives. One objective, the Heat Conservation Objective seeks to minimize the use of heat during the control of the environment. While this intuitively conflicts with the objective of maintaining the temperature above some fixed threshold expressed through the Lower Comfort Temperature Objective, the Controleum framework can handle such conflict using an approach combining the use of a Pareto frontier with a selection algorithm based on social welfare as described at the beginning of this section. The final objective, the Ventilation Conservation Objective, seeks to reduce the opening of the Variable Air Volume (VAV) damper position, to preserve overall energy consumption in the ventilation system. While this again potentially conflicts with the need to keep the $\mathrm{CO} 2$ level below a threshold as expressed by the $\mathrm{CO} 2$ Constraint Objective, this again is handled by Controleum using a Pareto frontier and social metric selection mechanism.

The objectives described above express their satisfaction towards a particular set of actuation points through minimization functions. Each objective contains a minimization function which is an implementation of the mathematical function that expresses the target of the objective.

The CO2 Constraint Objective considers the distance between the resulting state of the controlled zone (expressed as an output from the Zone Model) and a configurable target for $\mathrm{CO} 2$ in that particular zone. This distance equals the evaluation given by this objective to a particular solution. The greater the distance, the larger the violation of the CO2 target.

The Upper- and a Lower Comfort Temperature Objective considers the distance between the resulting temperature calculated by the Zone Model and an upper- and lower target temperature respectively. Again, the greater the distance from either of these objectives the greater the violation of the temperature band.

Finally, the Heat- and Ventilation Conservation Objectives consider the absolute consumption of heating and ventilation. For the Ventilation Conservation Objective, this is measured simply by adding the values of the actuator position for the VAV damper position, which is part of the solution suggested by the GA. This then assumes a linear relation between the VAV damper position and the energy consumption of the ventilation system. The Heat Conservation Objective returns an evaluation value which is the sum heat energy provided to the zone from heating sources, which corresponds to the sum of the output from "Qrad" in the Zone Model shown in Fig. 3. 


\section{Experimental CASE}

For our experiments, we consider a classroom in building OU44 at the University of Southern Denmark. The building is depicted in Fig. 4.

The construction of the building was completed in 2015 as an extension of the existing campus with its main purpose being teaching and offices.

The building constitutes part of the Smart Building Living Labs at the University of Southern Denmark and is used in ongoing research efforts to determine the optimal use of energy in buildings.

The building is equipped with a balanced ventilation system with 4 Air Handling Units (AHU) providing up to 35,000 m3/h of fresh air each. Each AHU serves approximately a quarter of the building and is equipped with two fans (supply and extract), a rotary wheel heat exchanger, and a heating coil. The ventilation air supply temperature is by default $21^{\circ} \mathrm{C}$. There is no mechanical cooling in the building. The fans operate to maintain constant pressures in the duct system. Each thermal zone is equipped with a VAV terminal. In the default control mode, the VAV dampers are controlled based on the indoor $\mathrm{CO} 2$ (maximum openness for $800 \mathrm{ppm}$ ). In addition, the building is equipped with a hydronic heating system. All rooms are equipped with radiators with BMS-controlled valves. The valve opening depends on the indoor temperature and the default temperature setpoint is $21^{\circ} \mathrm{C}$. The measured quantities include ventilation damper and radiator valve openings, indoor temperatures, indoor $\mathrm{CO}$, number of occupants (in selected rooms only), fan electricity consumption, and temperatures in the AHU. The AHU fans operate to maintain constant pressure in the ductwork, so the damper position in each room is correlated with the fan electricity consumption. If more dampers are open, the fans need to increase speed to maintain the pressure. Although this relationship is nonlinear, it is monotonous, so the damper position or room ventilation flow rate can be used as proxy objectives in the optimization. In this work, it is assumed that on a room level damper opening is linearly correlated with the room ventilation flow rate.

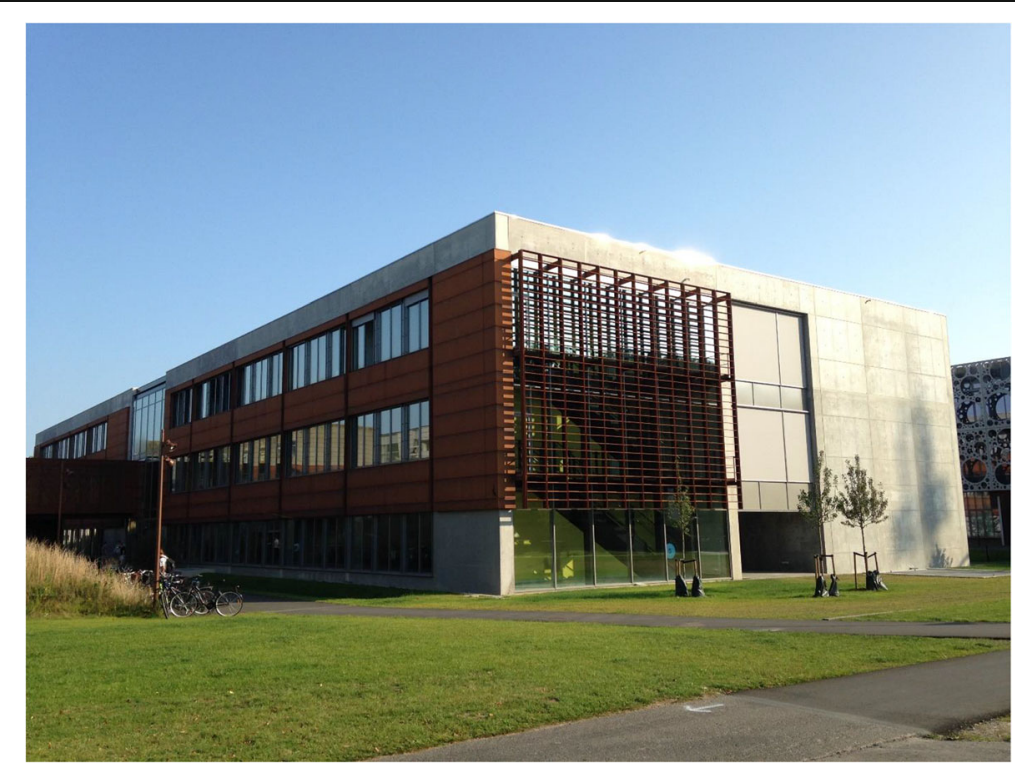

Fig. 4 Building OU44 at the University of Southern Denmark Odense Campus 
For a more detailed presentation of the Smart Building Living Lab at the University of Southern Denmark we refer to (Jradi et al. 2017a).

The experiments in this paper took place in the lecture Room U182 in OU44. The room is depicted in Fig. 5. The room has space for 84 occupants and has a size of 139 square meters.

\section{Configuration}

Reusability of the Digital Twin Framework is enabled through configuration. The configuration spans the configuration of the hardware interface, objective configuration, and model calibration.

In the following subsections, we go through the configuration points used to configure the digital twin used in these experiments.

\section{Interfacing towards sensors and actuators}

As the Zone Control Application accesses building instrumentation through sMAP, any data - be it sensors, actuators, or other data streams such as weather forecasts that the Zone Control Application needs access to, must be configured on sMAP. Drivers connected to SMAP are then responsible for reading from- or writing to external systems. For physical instrumentation, the sMAP instance that we are using has a driver to enable communication via OPC UA (Leitner and Mahnke 2006). For simulation purposes, proprietary drivers are used to communicate with the simulation model. From the perspective of the Zone Control Application there is no difference, as physical and virtual points appear similar on SMAP. This in turn promotes reusability, as configuring the Zone Control Application to run in real- vs simulated mode is merely a matter of configuring addresses for points on sMAP.

In a previous paper (Arendt et al. 2019) the Digital Twin Framework was configured to control a simulation model which comprises a digital building with 7 zones. Here

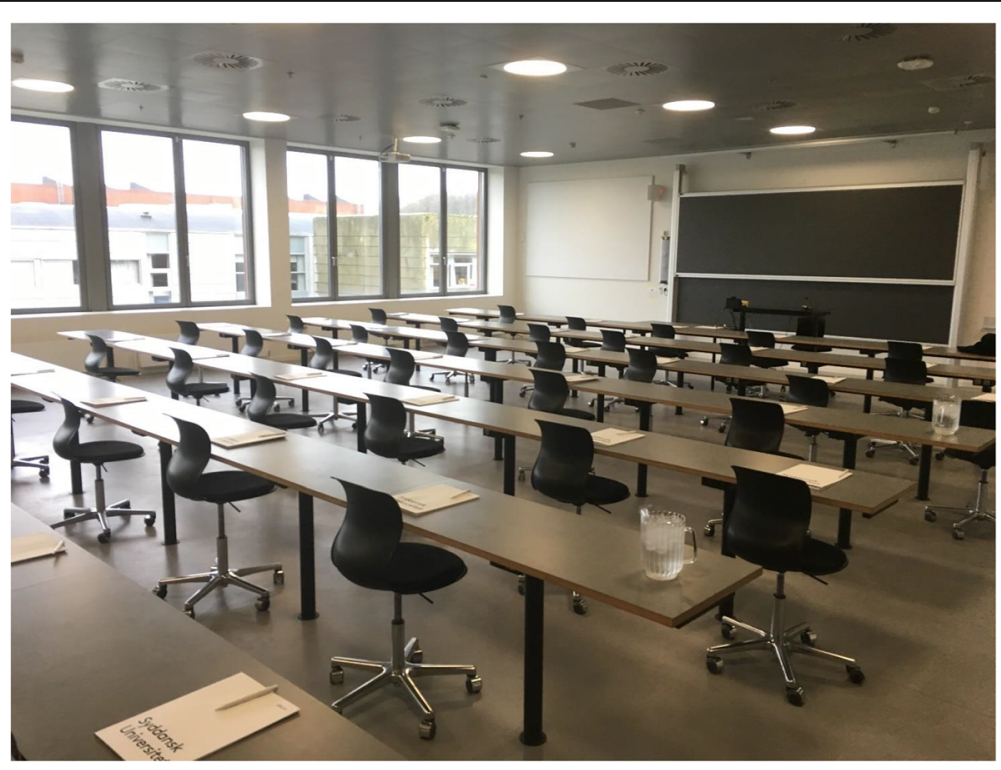

Fig. 5 Lecture room in building OU44 at University of Southern Denmark Campus Odense 
the purpose was to test the viability of different control strategies implemented in the digital twin framework as well as to test their performance compared to a standard rule-based control strategy. To this end the simulation models end points were mapped to sMAP and the corresponding sMAP addresses were configured in the Zone Control Application.

In this paper, the Digital Twin Framework was configured to control a physical room - the room U182 in building OU44 at the University of Southern Denmark, Campus Odense. To this end, a building management system, NetX BMS 2.0, was deployed and connected to the buildings KNX gateways. sMAP drivers then connected to NetX BMS 2.0 through an OPC UA interface which provided sMAP the ability to read from sensors and write to actuators in building OU44. The Zone Control Application was then configured with sMAP addresses pointing to physical hardware actuators and sensors installed in room U182 completing the link between the Digital Twin Framework and the physical instrumentation devices in room U182. Further, the Weather Driver in Fig. 1 was interfaced towards a weather station installed on building OU44 and the Xovis Camera Drivers were interfaced towards cameras deployed in room U182. The data from the Xovis Camera Drivers was supplied to OccuRE through sMAP, which in turn supplied occupancy prediction to sMAP making it available to the Zone Model.

\section{Objective configuration}

The objectives defined in the Zone Control Application express general goals in optimizing indoor comfort for human occupancy in an energy-efficient matter.

An instance-specific control strategy can be configured through parameters on these objectives.

The $\mathrm{CO} 2$ Constraint Objective enables the configuration of an upper limit for $\mathrm{CO} 2$. For the sake of the experiment in this paper, the CO2 limit was set at 900 PPM. While this is conservative concerning the 1000 PPM limit set forth by the Danish Working Environment Authority (Danish Working Environment Authority 2018) this limit is chosen to account for the fact that the generic Zone Model is likely to have some inaccuracy with respect to calculate the resulting environmental state of the controlled zone. The Lower- and Upper Comfort Temperature Objectives enable the configuration of a lower and upper-temperature target for the controlled environment. The values here are chosen based on the temperature span defined by the Danish Working Environment Authority (Danish Working Environment Authority 2018). This results in a lower temperature limit of $20^{\circ} \mathrm{C}$ and an upper-temperature limit of $23^{\circ} \mathrm{C}$.

\section{Zone model configuration}

The parameters needed to instantiate the Zone Model for U182 were found through normalized mean square error (NMSE) minimization, carried out using ModestPy (Arendt et al. 2018b) based on measurements collected in the period of 20-22 November 2018 as shown in Fig. 6. The measured data included indoor temperature, CO2, ventilation damper position, radiator valve position, number of occupants, outdoor temperature, and solar radiation. Ten parameters in total were calibrated: (1) infiltration rate, (2) solar heat gain coefficient, (3) indoor air thermal mass, (4) internal walls thermal mass, (5) external walls thermal resistance, (6) internal walls thermal 


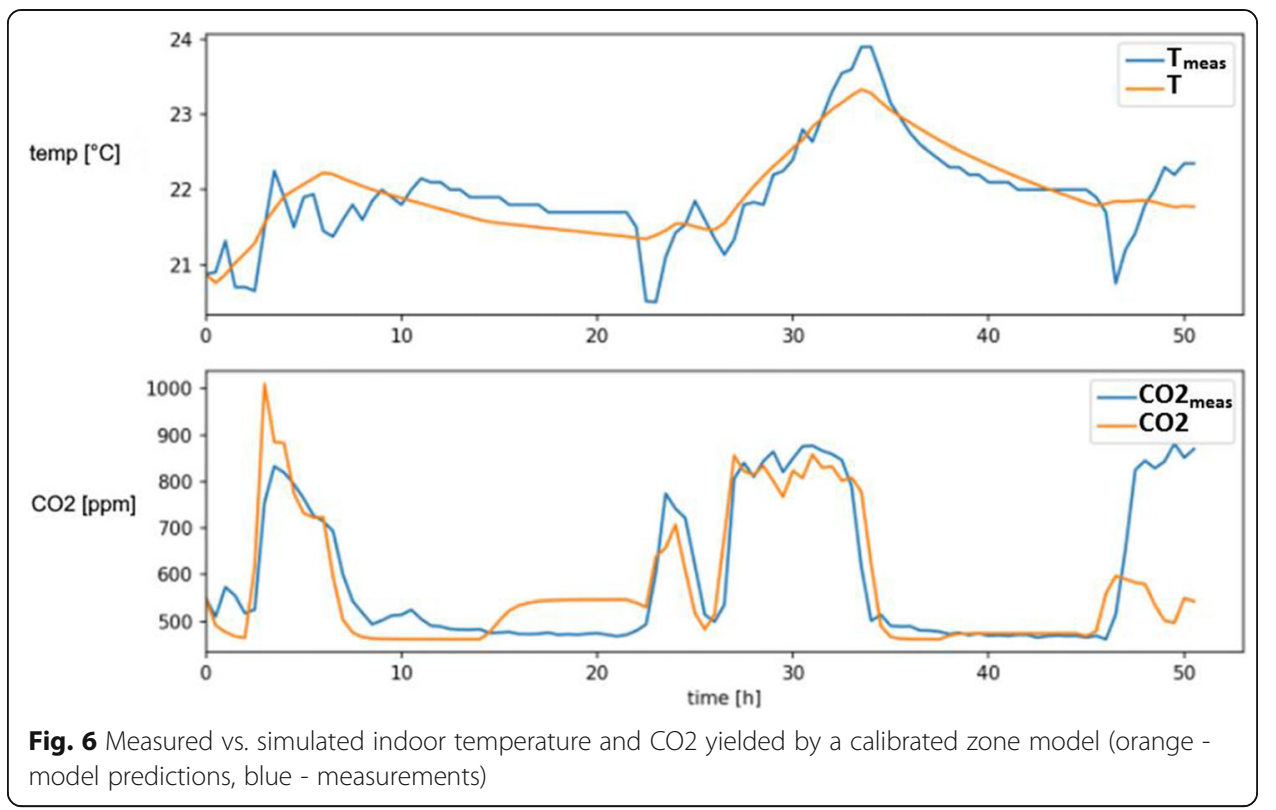

resistance, (7) occupant heat generation scaling factor, (8) ventilation supply air temperature, (9) maximum ventilation flow rate (system capacity), and (10) CO2 generation rate per person.

\section{Experiments}

The goal of the experiments was to demonstrate that the proposed method applying MPC to building automation can maintain comfort levels and to identify the potential for energy savings. To evaluate this, we observed the temperature and the $\mathrm{CO} 2$ levels in room U182 of building OU44 at the University of Southern Denmark, Campus Odense. We observed baseline measurements from one day of default operation and used the Digital Twin Framework to control the environment in room U182 during a single day of operation as well.

\section{Baseline}

During default operation, rule-based control is used with (static) $\mathrm{CO} 2$ thresholds and hysteresis to control the VAV damper position. Specifically, the rule-based control opts for 1 of three settings for VAV damper opening: At a CO2 level of 750 PPM or less the VAV damper is $45 \%$ open, between 750 PPM and 900 PPM the VAV damper opening is set to $70 \%$ and above 900 PPM the VAV damper opening is $100 \%$. This mode of operation uses a hysteresis of 100 PPM when CO2 levels are dropping (to avoid rapid switches around the thresholds). Figure 7 shows the CO2 levels and the VAV positions during hours of operation on October 24th. As seen, the two graphs have a high degree of correlation, and the maximum CO2 level is 1046 PPM which is above the threshold of 1000 PPM described by the Danish Working Environment Authority (Danish Working Environment Authority 2018). However, it is worth noting that this only happens in a very short period and that the ventilation actuation immediately reacts to the increase in the $\mathrm{CO} 2$ level. Worth noting from the figure is also that the VAV position is 


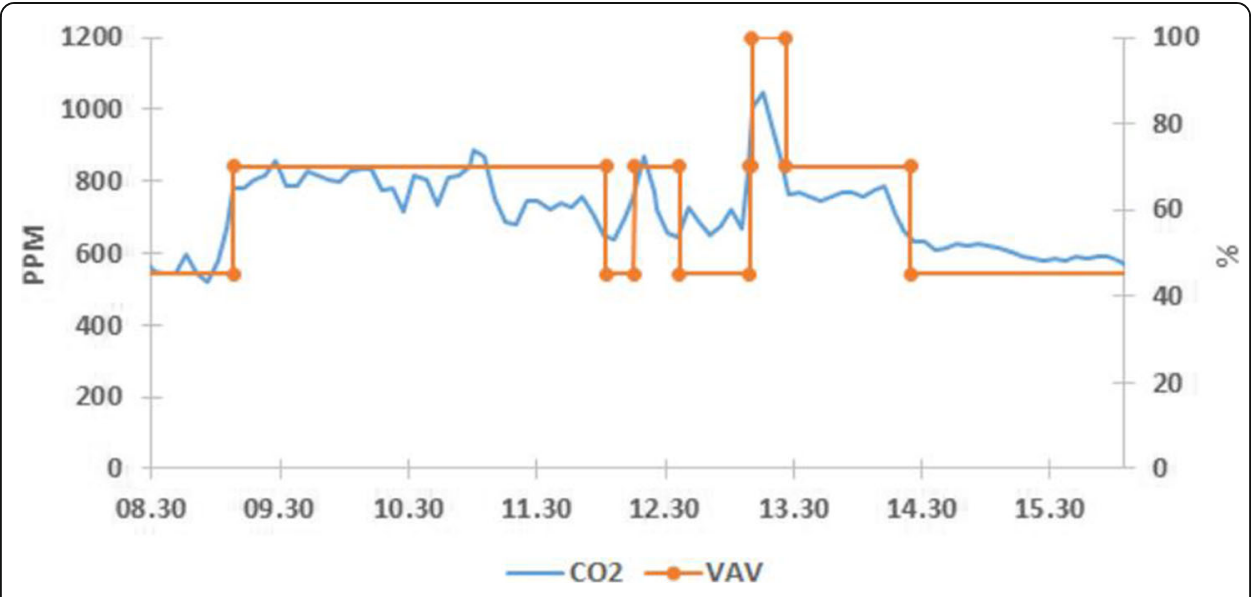

Fig. 7 CO2 levels and VAV damper position observed on October 24th, 2018 during default operation

always above $40 \%$ during hours of operation. This is also the case during periods in which ventilation has little or no effect, as can be seen from around 14:30. With no ways of predicting occupation built into the default operation strategy, this is the only way to ensure that the air quality of a room is ventilated sufficiently low to not exceed thresholds when a room is suddenly occupied, as the actuation of the VAV position is reactive.

The rule-based control applies a fixed setpoint of $21^{\circ} \mathrm{C}$ for the heating of the zone during hours of operation.

As can be seen from Fig. 8 the recommended temperature span between $20^{\circ} \mathrm{C}$ and $22{ }^{\circ} \mathrm{C}$ is violated most of the day. It should be noted that Danish buildings do not have air condition (AC) to suppress high temperatures. Ventilation systems can be used to a degree, but they do not have the capacity of a dedicated AC system which is also reflected by the temperature levels in Fig. 8 .

Based on these observations, the goal for control using the Zone Control Application is that CO2 levels should be mostly below the threshold of 1000 PPM described by the Danish Working Environment Authority (Danish Working Environment Authority 2018) but occasional swings above this level can be expected as was seen during default operation. This is due to the limitations in the capacity of the installed ventilation. We

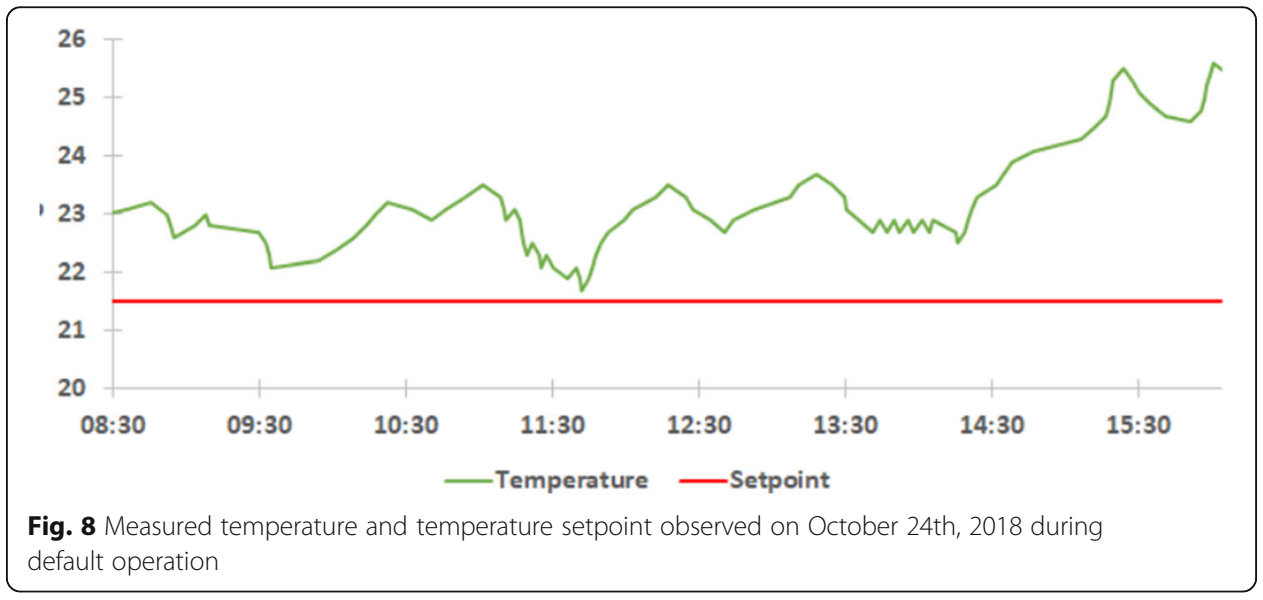


also expect the Zone Control Application to use a more energy-efficient strategy concerning ventilation, as occupancy is now predicted, and energy-conserving objectives are in place.

\section{Digital twin control experiment}

On December 14th, 2018, the Digital Twin Framework was put in operation effectively taking over control of the actuators in room U182 between 8:30 and 16:00 on that day. This day was a normal day of operation meaning that the room was in normal use throughout the experiment. The participants in the room were unaware of the conducted experiment and thus unable to actively influence the results.

Figure 9 shows the VAV damper position levels and the $\mathrm{CO} 2$ levels measured throughout the day. We see that the CO2 level is maintained below 1000 PPM for most of the day, except for a narrow time frame around 15:30. Comparing these results with the results during normal rule-based control in Fig. 7 we see that the amount of violation concerning the $\mathrm{CO} 2$ threshold is comparable between the two approaches.

Figure 9 also shows that the Zone Control Application uses a more fined grained control of the VAV actuator when compared to the VAV damper positions shown in Fig. 7. Aside from this, the Zone Control Application also opts for a complete shutdown of the ventilation during hours of operation when no need for ventilation is predicted by the algorithm. This can be seen in the timespan between 09:06 and 10:06 where the VAV damper position setpoint is observed to be $0 \%$. This differentiates from the pattern seen in Fig. 7 where the VAV damper position setpoint is always above 40\% during hours of operation.

Concerning temperatures, we see from Fig. 10 that the Zone Control Application alters the temperature setpoint as opposed to the default operation where a static temperature setpoint is used.

By comparing Fig. 8 and Fig. 10 we observe that the temperature thresholds are less violated during the experiments where the Zone Control Application is in operation. At this point, it should be noted that the indoor temperature depends on a multitude of variables aside from the applied control strategy. These variables include outdoorand adjacent temperatures as well as occupancy.

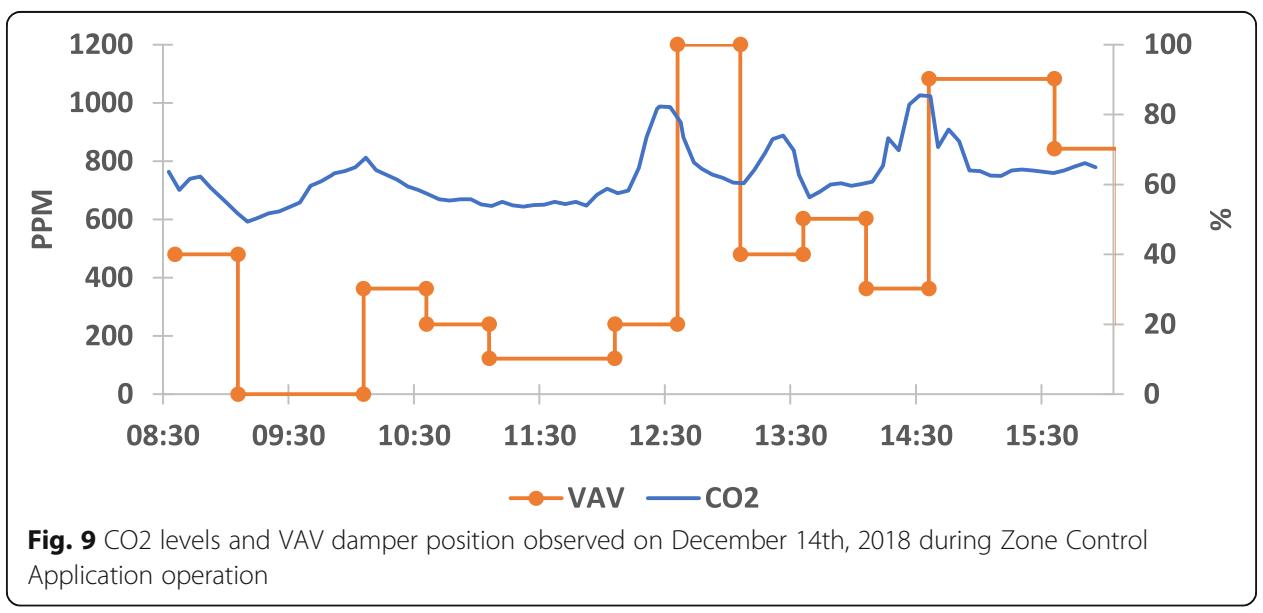




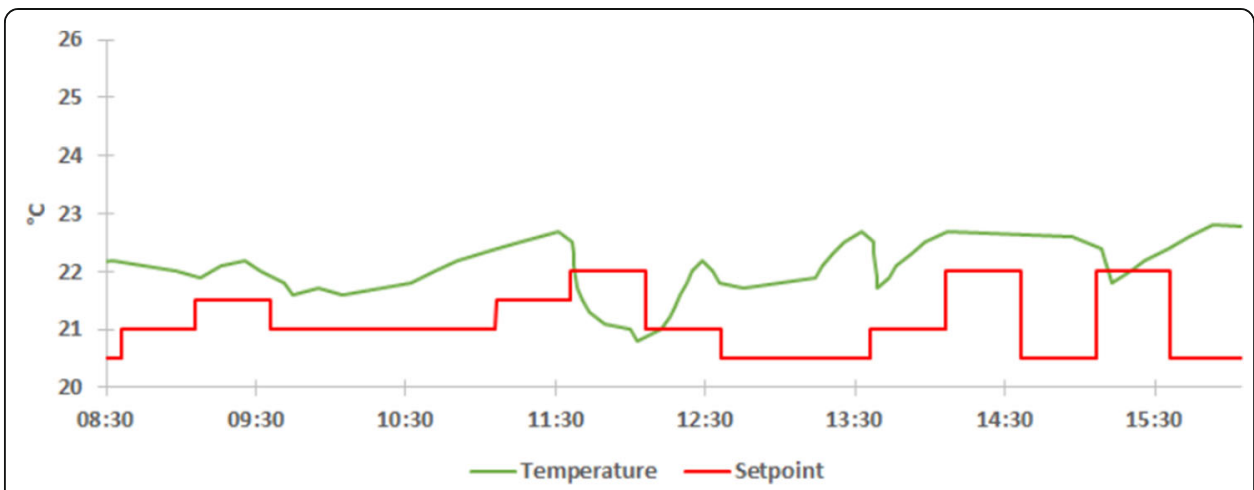

Fig. 10 Temperature and temperature setpoints observed on December 14th, 2018 during Zone Control Application operation

\section{Discussion}

Using the Zone Control Application developed in the Digital Twin Framework we achieved overall comfort levels which were comparable to those achieved by the rulebased control strategy employed during default operation.

While the Zone Control Application did violate the $\mathrm{CO} 2$ threshold for a short time span, as shown in Fig. 9, the same was observed during default operation as seen from Fig. 7. Analyzing the data of the $\mathrm{CO} 2$ levels versus the VAV damper setpoints applied by the Zone Control Application we see that the algorithm behaves reactively in cases where it observes that the $\mathrm{CO} 2$ level is rising beyond comfort levels. Here it seems that the predictions made by OccuRE, in this case, were conservative with respect to the actual population in the zone. Indeed, by observing the predictions made by OccuRE in Fig. 11 we see that the number of anticipated persons in the room is somewhat flat throughout the day which does not map to the fluctuations in $\mathrm{CO} 2$ levels that we see from Fig. 9.

The Zone Control Application can counteract the inaccuracy in the prediction through a reactive behavior, as the $\mathrm{CO} 2$ Constraint Objective uses the output of the Zone Model to decide if a VAV damper position setpoint is suitable. The Zone Model in turn considers not only the predicted number of occupants but also the current state of the controllable zone as well as the VAV damper position setpoint to calculate a resulting state for U182. Given a high initial $\mathrm{CO} 2$ level this means that the CO2

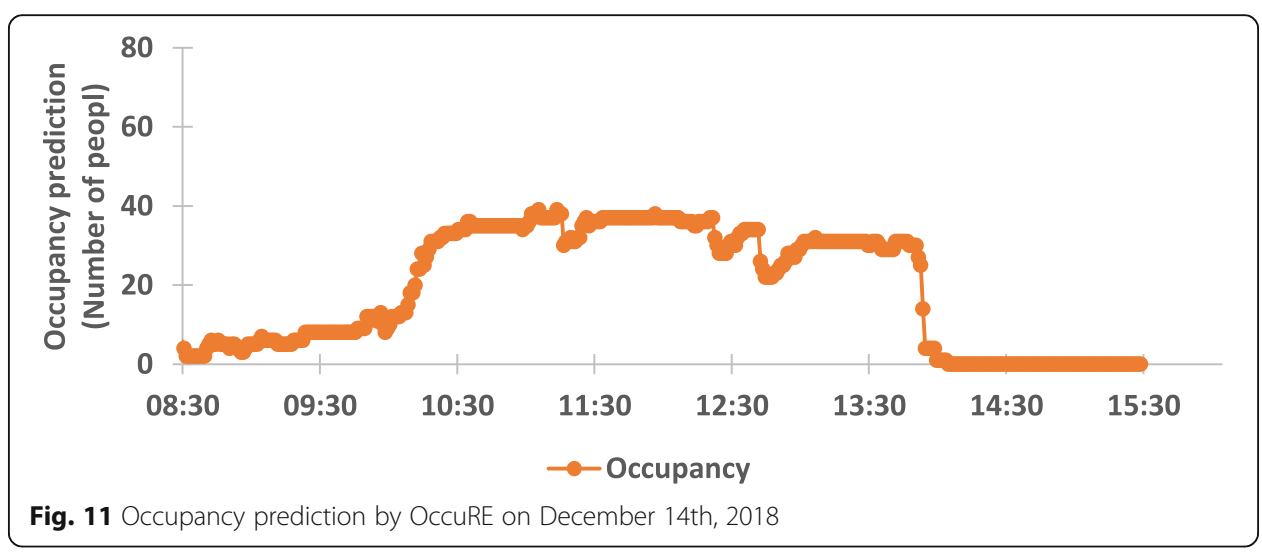


Constrain Objective is going to prefer a higher VAV damper position threshold to force the $\mathrm{CO} 2$ state of $\mathrm{U} 182$ below threshold values.

Figure 9 shows that the Zone Control Application uses a fine-grained control of the VAV actuator as the VAV damper position setpoint is varied in $10 \%$ steps between 0 and $100 \%$ during the day. The default operation shown in Fig. 7 values of $45 \%, 70 \%$, and $100 \%$ are observed.

As the default operation relies entirely on a reactive behavior, a default setpoint of $45 \%$ to ensure that the $\mathrm{CO} 2$ levels do not overshoot the defined comfort levels too much before the system can act. The Zone Control Application on the other hand can predict active use of the room and as a result, will pre-emptively ventilate before an anticipated use of the room. With accurate predictions of room usage, this means that the Zone Control Application can operate more energy efficiently, as ventilation is only applied when needed. However, this paper cannot quantify the energy savings achieved through this strategy due to several reasons. For one, natural variation in weather and usage of the room needs to be accounted for. This can be done by expanding the experiments to run for a year and make a subsequent comparison with a year of default operation data. Further, the energy use from the ventilation system is aggregated across the entire building. Hence, to get a direct comparison in energy consumption levels, the strategy needs to be applied to all rooms in building OU44. However, an approximation based on estimated power consumption per room as a function of VAV damper opening and the room size could likely give a rough idea of the energy-saving potential.

It is worth noting that by using the MPC strategy implemented by the Zone Control Application, a possibility exists to avoid any compromises on the $\mathrm{CO} 2$ threshold. Although Fig. 9 showed a minor violation of the $\mathrm{CO} 2$ threshold, this could likely be avoided if the occupancy prediction is improved.

With respect to temperature, it is harder to draw hard conclusions. Although we observed better comfort concerning temperature during Zone Control Application operation, we also note that the indoor temperature is a product of more than the actuation of thermostats and the VAV damper position. As buildings in Denmark are not equipped with dedicated HVAC solutions to cool the indoor climate, the task of cooling rooms is delegated to the ventilation system. However, the ventilation system is not dimensioned to guarantee that this threshold can be adhered to under all circumstances. This means that if a high outdoor temperature is combined with a high number of occupants in the room, the ventilation system in U182 is simply unable to keep the temperature sufficiently low. Thus, while the use of the Zone Control Application does not seem to violate the comfort temperature limits, we cannot decisively say that the Zone Control Application outperforms the rule-based approach used during default operation.

\section{Conclusion}

This paper presents a Digital Twin Framework for performing MPC on physical- and simulated buildings.

The Digital Twin Framework constitutes an architecture describing applications for building control, occupancy prediction, and a common data format implementation based on sMAP. The Digital Twin Framework contains a parametrized Zone Model which can be calibrated against (virtual or physical) controllable zones as well as a 
parametrized Zone Control Application which enables the configuration of targets for temperature- and $\mathrm{CO} 2$.

A specific instance of the Digital Twin Framework was created to perform control of a classroom in room U182 of building OU44 at the University of Southern Denmark, Campus Odense. Here experiments have shown that the proposed system can provide comfort levels comparable to those maintained by the existing control strategies implemented by a commercial building management system while enabling the implementation of strategies to improve energy efficiency. This is done by solving a multi-objective optimization problem that defines objectives for comfort as well as energy (heat- and electricity) conservation inside the Zone Control Application. The implementation showed potential for improved energy efficiency, as the VAV damper position was reduced to zero when no occupancy was predicted in the immediate future. This behavior was an improvement over the default rule-based strategy which maintains the VAV damper position at $45 \%$ through all hours of operation to avoid any severe violation of $\mathrm{CO} 2$ thresholds when occupants arrive at the room. However, it is currently impossible to quantify the potential energy savings due to the nature of the ventilation system which spans all rooms in building OU44, and the natural fluctuation in weather and use of the room.

\section{Abbreviations}

AHU: Air handling units; GA: Genetic algorithm; MPC: Model predictive control; NMSE: Normalized mean square error; VAV: Variable air volume

\section{Acknowledgements}

Not applicable.

\section{About this supplement}

This article has been published as part of Energy Informatics Volume 4, Supplement 2 2021: Proceedings of the Energy Informatics.Academy Conference Asia 2021. The full contents of the supplement are available at https:// energyinformatics.springeropen.com/articles/supplements/volume-4-supplement-2.

Funding (for both the study and the publication fees)

The paper was part of the research project: COORDICY - ICT-driven Coordination for Reaching 2020 Energy Efficiency Goals in Public and Commercial Buildings, funded by Innovation Fund Denmark.

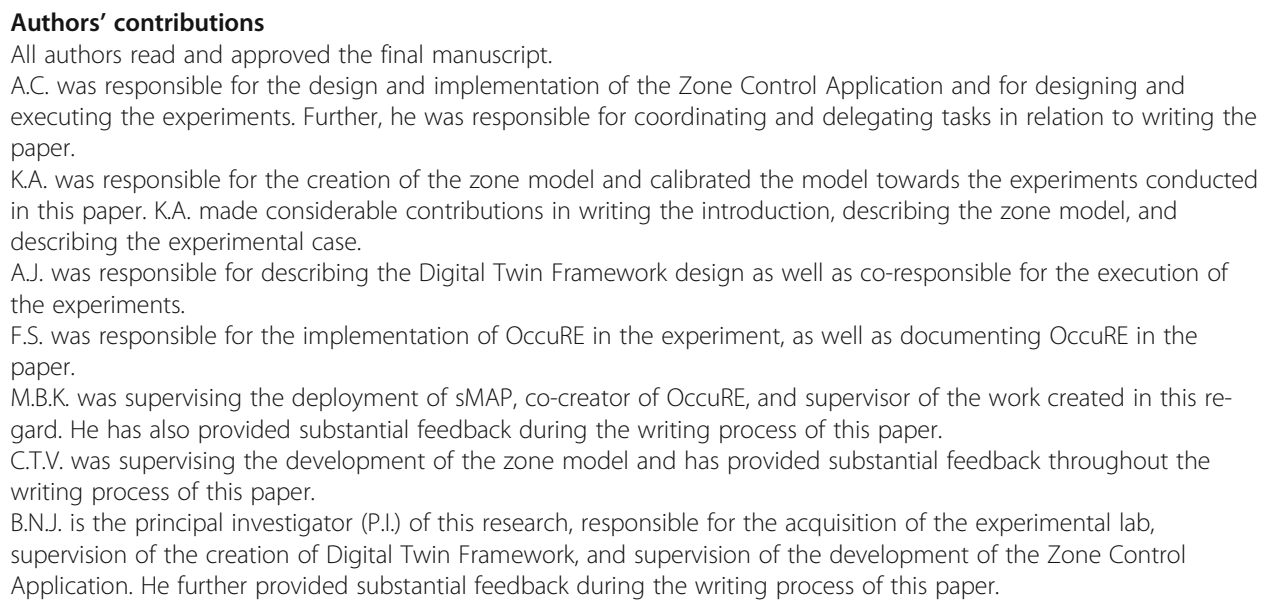




\section{Declarations}

\section{Ethics approval and consent to participate}

Not applicable.

\section{Consent for publication}

Not applicable.

\section{Competing interests}

The authors declare that they have no competing interests.

\section{Author details}

${ }^{1}$ Center for Energy Informatics, Maersk Mc-Kinney Moller Institute, University of Southern Denmark, Campusvej 55, 5230 Odense M, Denmark. ${ }^{2}$ Software engineering unit, Maersk Mc-Kinney Moller Institute, University of Southern Denmark, Campusvej 55, 5230 Odense M, Denmark.

Published: 24 September 2021

\section{References}

Al-Ghaili AM, Kasim H, Al-Hada NM, Jørgensen BN, Othman M, Jihua W (2021) Energy Management Systems and Strategies in Buildings Sector: A Scoping Review. IEEE Access 9:63790-63813

Arendt K, Clausen A, Mattera CG, Jradi M, Johansen A, Veje C et al (2019) Multi-objective model predictive control framework for buildings. In: Procedings of the 16th IBPSA international conference and exhibition building simulation 2019, pp 822-829

Arendt K, Jradi M, Shaker HR, Veje C. Comparative analysis of white-, gray-and black-box models for thermal simulation of indoor environment: Teaching building case study. In Proceedings of the 2018 Building Performance Modeling Conference and SimBuild co-organized by ASHRAE and IBPSA-USA, Chicago 2018a. p. 26-28.

Arendt K, Jradi M, Wetter M, Veje CT (2018b) ModestPy: An Open-Source Python Tool for Parameter Estimation in Functional Mock-up Units. In: Proceedings of The American Modelica Conference 2018, October 9-10, Somberg Conference Center, Cambridge MA, USA. Linköping University Electronic Press, Linköpings universitet, pp 121-130

Bianchini G, Casini M, Vicino A, Zarrilli D (2016) Demand-response in building heating systems: A Model Predictive Contro approach. Appl Energy 168:159-170

Billanes JD, Ma Z, Jørgensen BN (2017) Consumer Central Energy Flexibility in Office Buildings. J Energ Power Eng 11

Billanes JD, Ma Z, Jørgensen BN (2018) The Bright Green Hospitals Case Studies of Hospitals' Energy Efficiency And Flexibility in Philippines. In: 2018 8th International Conference on Power and Energy Systems (ICPES), pp 190-195

Cao X, Dai X, Liu J (2016a) Building energy-consumption status worldwide and the state-of-the-art technologies for zeroenergy buildings during the past decade. Energy Build:198-213

Clausen A, Umair A, Demazeau Y, Jørgensen BN (2020a) Impact of Social Welfare Metrics on Energy Allocation in MultiObjective Optimization. Energies. 13

Danish Working Environment Authority. Indeklima (Danish). 2018. [Online; accessed March-2021].

Dawson-Haggerty S, Jiang X, Tolle G, Ortiz J, Culler D (2010) sMAP: A Simple Measurement and Actuation Profile for Physical Information. In: Proceedings of the 8th ACM Conference on Embedded Networked Sensor Systems. Association for Computing Machinery, New York, pp 197-210

Dawson-Haggerty S, Krioukov A, Taneja J, Karandikar S, Fierro G, Kitaev N et al (2013) BOSS: Building Operating System Services. In: 10th USENIX Symposium on Networked Systems Design and Implementation (NSDI 13). USENIX Association, Lombard, pp 443-457

De Coninck R, Helsen L (2016a) Practical implementation and evaluation of model predictive control for an office building in Brussels. Ener Build:290-298

Dong B, Lam KP (2014) A real-time model predictive control for building heating and cooling systems based on the occupancy behavior pattern detection and local weather forecasting. Build Simul:89-106

Drgoňa J, Arroyo J, Figueroa IC, Blum D, Arendt K, Kim D et al (2020) All you need to know about model predictive control for buildings. Annu Rev Control 50:190-232

Garcia CE, Prett DM, Morari M (1989a) Model predictive control: Theory and practice-A survey. Automatica:335-348

Jørgensen BN, Kjærgaard MB, Lazarova-Molnar S, Shaker HR, Veje CT (2015) Challenge: Advancing Energy Informatics to Enable Assessable Improvements of Energy Performance in Buildings. Association for Computing Machinery, New York, pp 77-82

Jradi M, Sangogboye FC, Mattera CG, Kjærgaard MB, Veje C, Jørgensen BN (2017a) A world class energy efficient university building by danish 2020 standards. Energy Procedia 132:21-26

Jradi M, Veje C, Jørgensen BN (2017b) Deep energy renovation of the Mærsk office building in Denmark using a holistic design approach. Ener Build 151:306-319

Kjærgaard MB, Arendt K, Clausen A, Johansen A, Jradi M, Jørgensen BN et al (2016a) Demand response in commercia buildings with an Assessable impact on occupant comfort. In: 2016 IEEE International Conference on Smart Grid Communications (SmartGridComm), pp 447-452

Kjærgaard MB, Johansen A, Sangogboye F, Holmegaard E (2016b) Occure: an occupancy reasoning platform for occupancydriven applications. In: 2016 19th International ACM SIGSOFT Symposium on Component-Based Software Engineering (CBSE), pp 39-48

Lazarova-Molnar S, Kjærgaard MB, Shaker HR, Jørgensen BN (2015) Commercial buildings energy performance within context occupants in spotlight. In: 2015 International Conference on Smart Cities and Green ICT Systems (SMARTGREENS), pp 1-7

Leitner SH, Mahnke W (2006) OPC UA-service-oriented architecture for industrial applications. ABB Corp Res Center 48:61-66

Li X, Malkawi A (2016a) Multi-objective optimization for thermal mass model predictive control in small and medium size commercial buildings under summer weather conditions. Energy:1194-1206 
Ma Z, Badi A, Jørgensen BN (2016) Market opportunities and barriers for smart buildings. In: 2016 IEEE Green Energy and Systems Conference (IGSEC), pp 1-6

Ma Z, Billanes JD, Jørgensen BN (2017b) Aggregation Potentials for Buildings—Business Models of Demand Response and Virtual Power Plants. Energies. 10

Ma Z, Dalmacio Billanes J, Kjærgaard MB, Jorgensen BN (2017a) Energy flexibility in retail buildings: From a business ecosystem perspective. In: 2017 14th International Conference on the European Energy Market (EEM), pp 1-6

Marler RT, Arora JS (2004) Survey of multi-objective optimization methods for engineering. Struct Multidiscip Optim 26:369-395 Sangogboye FC, Arendt K, Singh A, Veje CT, Kjærgaard MB, Jørgensen BN (2017) Performance comparison of occupancy count estimation and prediction with common versus dedicated sensors for building model predictive control. In: Building Simulation, pp 829-843

Sørensen JC, Jørgensen BN (2017) An extensible component-based multi-objective evolutionary algorithm framework. In: Proceedings of the 6th International Conference on Software and Computer Applications, pp 191-197

\section{Publisher's Note}

Springer Nature remains neutral with regard to jurisdictional claims in published maps and institutional affiliations.

Submit your manuscript to a SpringerOpen ${ }^{\odot}$ journal and benefit from:

- Convenient online submission

Rigorous peer review

- Open access: articles freely available online

- High visibility within the field

- Retaining the copyright to your article 